\title{
Design Perspectives: Sampling User Research for Concept Development
}

\author{
Petri Mannonen and Mika P. Nieminen \\ Helsinki University of Technology, Department of Computer Science and Engineering, \\ P.O. Box 9210, 02015 TKK, \\ Finland \\ \{petri.mannonen, mika.nieminen\}atkk.fi
}

\begin{abstract}
In user-centered design user research usually produces detailed description of the users, analysis of relevant actions and the specifics of the environment and artefacts thereof. However additional step of sampling from different viewpoints is required to more efficiently kick-start user-centered product concept development. Design perspectives, abstracted cross-category samples of user research results, are introduced as means to increase the usefulness of analyzed data during user-centered product concept development. Defining additional layer of entities in addition to conventional user research results helps to migrate from the pedant data-gathering phase to the more creative concept development phase. This paper describes definitions of entities involved in constructing design perspectives in a user research method independent manner with examples from a project developing new concepts for mobile and distributed team work.
\end{abstract}

Keywords: User research, user-centered design, concept development, data analysis, process development.

\section{Introduction}

User-centered design is based on the idea of developing products and services that support the user's tasks in the context she operates in. This goal is approached by first understanding the context of use and the requirements of the users, and then making the design decisions based on that understanding. User research is the phase in usercentered design process where the knowledge of the users' tasks and environment is gathered [1].

\subsection{User Research}

The aim of user research is to provide sufficient understanding of the users and the context of use in order to be able to make and justify the design decisions. ISO 13407 standard describes user research as understanding and specifying the context of use. The context is defined as the characteristics of the users, tasks and the organizational and physical environment. The result of user research is typically a description document. User and organizational requirements for the product are formed based on 
the context of use description. However, the standard does not describe the methods used in the user research. It only suggests that the whole process should be iterative and that the description document should evolve during the whole development project [1].

Aiming for adequate understanding is the key difference between product development project's user research and traditional qualitative research, for instance anthropological studies. The main reason for this difference is the limited amount of time available for conducting the research [2]. This has lead to development of specialized research methods to gather specific type of information or to hasten the traditional qualitative research methods. For instance cultural probes focus on the personal feelings and experiences of the users [3], while contextual inquiry emphasizes the context in which work is performed [4]. The basic methodology described in the literature includes observations, interviews and questionnaires, from which a myriad of variations have sprung to tackle more specialized needs (for examples please refer to $[4,5,6])$.

User research methods descriptions usually give accurate and sometimes even practical step-by-step instructions on how to conduct the data gathering and what are the strengths of the method compared to the other user research methods. Although the research methods for gathering user and context of use information are numerous they rarely include exact instructions or suggestions how to analyze the gathered data. The authors mostly only provide general examples or rough guidelines for the data analysis. In the literature it is widely agreed that multidisciplinary processes, highly visual methods and attending to projects' special needs produce best results in user data analysis $[2,4,6]$.

In qualitative research the data analysis is depicted as continuous and somewhat iterative process during which the researcher digests vast amount of information and builds up a deep understanding of the studied phenomena trough scrupulous analysis of each individual piece of data available [7]. The analysis process begins and ends with going trough the whole gathered data. At the beginning the motivation is to understand the amount of the data as well as the main themes, while at the end the results are evaluated against the original data $[7,8]$. Fetterman describes the building of holistic understanding as crystallization in which the researcher condenses the information to elegant descriptions [9].

Product development is limited by availability of resources, calendar time and human resources, also during the data analysis. After the data has been gathered as efficiently as possible also the analysis must be done in rapid succession. In many cases the long analysis phase followed by a crystallization of the researchers understanding is too time-consuming for product development purposes.

Based on the themes of the ISO 13407 standard most user research results are reported with document(s) describing the user (profiles, personas, user group and role definitions, etc), environment (Environment lists, artefact lists, etc.), users' actions (task models and sequences, scenarios, etc.) and requirements derived from the preceding descriptions [4,5,6]. The usefulness of having several different representations and point of views is emphasized as in many cases it is difficult to predict exactly what sort of information is needed in the following product development phases. 
Though the aim is to produce a holistic view of the users and their contexts, usually the deliverables tend to report the results as separate descriptions from predefined viewpoints (user, environment and actions).

From the viewpoint of concept development i.e. when using the user research data to create new product concepts, there seems to be a need to take the analysis a step further or at least restructure the results differently. The following chapter describes some of the special characteristics of concept development that suggest that while requirements drawn from the user research are suitable for developing predefined products, they tend to restrict the creation of new product innovations.

\subsection{Characteristics of Concept Development}

User-centered concept development can be described as early phase exploratory process in product development aiming to create new product innovations [10]. The motivation for user-centeredness is to introduce information about real users to the product development cycle as early as possible. Injection of user understanding to the process enables development of product ideas that respond more accurately to users' needs and comply with the context of use of the studied domain. From the company's perspective user research can also point out new product potential or new customer groups for their overall development outside the then current project. The main phases of user-centered concept development process can be outlined to include: 1) project commitment, 2) user and technology research, 3) innovation sprint, 4) concept creation and validation, and 5) project assessment. The final deliverables of this kind of process are concept descriptions detailed enough to support decision making whether to start the actual product development project or to discard the concept. [11]

From the perspective of the project team the concept development project includes three separate modes of working. During the project commitment and user and technology research one should act objectively and analytically. The idea generation phase, or innovation sprint, requires the team to be able to produce innovative and even surprising product ideas. Concept creation and validation and project assessment are a more concrete phase during which the produced ideas are evaluated and developed further and finally the whole project is wrapped up for easy adoption in the actual product development.

After user research the process' working mode changes (or more accurately is brutally rushed away) from analytical and objective research point of view to more innovative idea generation [11]. The analytical researcher's and the innovative designer's points of view are profoundly different even in situations where one and same team conducts the whole concept development process. The researcher tries to objectively understand and describe the object of research and the designer inevitability aims towards changing the world she is designing new products to.

Pre-existing know-how and roles affect on how the world and events around us are interpreted. Same subjects and events manifest themselves from different point of view based on the role of the actor (e.g. developer, evaluator, user, customer). The different points of views and frames of reference can easily lead to conflicts and misunderstandings between people. Schön claims that one way of solving these conflicts is to develop new point of view which is shared between the conflict parties or at least understood by them, hence sometimes referred as problem framing. The shared frames of reference enable the parties to work towards shared goals together [12]. 
In concept development projects conflicts and misunderstandings can be seen inside the concept development project in the beginning of the idea generation where in many cases there is a strong need for concrete starting points and the comprehensive user and environment descriptions seem to offer too little gripping surface for successful creative leap. The explanatory and objective user research results can make everything seem equally interesting and important.

After the innovation phase when further developing and defining the concept ideas the project team faces many decisions, which have to be based on the knowledge of users and their context of use. In decision-making and evaluation the project team has to be objective and nonbiased. Too subjective or one-sided information complicates the decision-making. The user research results have to support the objective evaluation of the concept candidates.

These above mentioned characteristics of product and concept development are in some extent noticed by user-centered product development literature. Often suggested solution to the challenges is to produce multiple models or other presentations of the findings [4]. This inevitably leads to heavy and time-consuming processes. Another solution is to start the idea generation phase (design phase) by collecting team's shared visions and using them as input for further idea generation [4]. This working method risks compromising the traceability of the process as the visions are not always based on user research findings and can embody team's prejudices.

The above concept development process sets some specific requirements for both the implementation of user research and especially for the deliverables of the research phase.

- The user research process should be as transparent as possible. Especially concept validation would gain if each individual interpretation/decision could be traced to the original observation or phenomenon.

- The deliverables should be presented in easily adoptable and understandable, preferably visual, form to minimize the time required to absorb the knowledge and to avoid false conclusions.

- The deliverables should depict the users and their tasks and contexts truthfully and comprehensively, thus enabling a solid ground for the following creative process.

- The deliverables should support the product development team while its working mode changes.

- Both the process and the deliverables should cater the overall goals of the project and the customer (i.e. maximize the utilization of earlier acquired know-how and support corporate vision).

In this paper a new abstraction, design perspective, is proposed to increase the usefulness of the findings of user research and therefore provide better starting point for concept development than current reporting practices.

Design perspectives and proposed analysis framework steers the analysis and presentations of the outcomes towards more concise concept development while balancing the user-centered approach with project and company specific development drivers in an efficient and light-weight manner. 


\section{Definitions}

Although analyses of different qualitative data sets are different there are common features [7]. In addition of the general process of proceeding from individual observations towards more holistic view and verifying the findings with the original data also the entities evolving during the user research data analysis are similar at an abstract level.

The analysis framework depicted in figure 1 shows the main entities involved in user research data analysis from observed phenomena to categories and finally to design perspectives. The framework can be seen as hierarchical and cross-connected organization of the collected data. In the following chapters each of these entities are defined followed by a description of the process leading to design perspectives with examples.

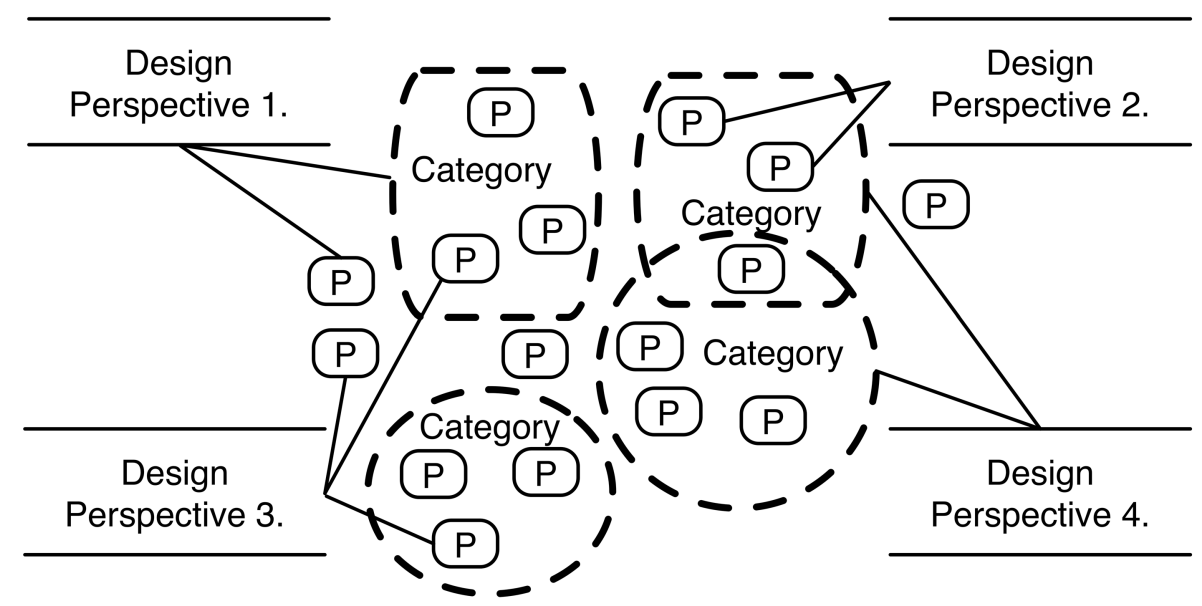

Fig. 1. User research data analysis framework

\subsection{Phenomena}

The phenomena (shown as ' $\mathrm{P}$ ' in figure 1) are observable facts or events within the user research data. A phenomenon can be based on a single observation or be a combination of several supporting observations. Phenomena do not group the observations arbitrary together, but are more like labels for relevant observations or surfaced observation groups. However, the phenomena are not standard-sized or necessary comparable with each other. Usually the concept development project has one or more focus areas or interests. Thus the information gathered in the user research is deeper in some areas and more general in others.

\subsection{Categories}

During the analysis associative methods are used to form groups of phenomena hereinafter called categories. Unlike the hierarchies in affinity diagrams with all the 
observations built into a single three with a top node, the categories do not need to have an existing or fabricated uniting higher level parent. Categories group together deeply linked phenomena and they may nest other categories. In figure 1 categories are marked out with dash lines.

The goals of user research (and the user-centered process) steers the analyzing process towards 'standardized' structure of user, environment and context of use related information. Additionally the projects' special goals usually show up in categorization. E.g. In a project studying distributed and mobile knowledge work the analysis of user research data produced separate categories for enablers and disablers of mobility and distributedness.

Categories and phenomena form the backbone of the analysis process leading to design perspectives. All the details about the users, environments and observed actions are included in them. Accurate documentation of the categories and phenomena enable traceability of the analysis as categories consist of phenomena and a phenomenon is directly linked to the actual observed facts or events.

\subsection{Design Perspectives}

Design perspectives are abstracted cross-category samples of user research results outlining discovered potential contradictions, widely visible tendencies and interesting possibilities. Design perspectives emphasize the interpretations and viewpoints of the users and the user research taskforce allowing them to contribute the tacit knowledge that could otherwise be omitted as it may fall outside the conventional reporting practices. From the concept development point of view design perspectives are a tool to kick-start the idea generation phase. They depict the user research results from different viewpoint augmenting the analyst's results with the individual user's or designer's point of view.

Creation of design perspectives is an extension of user research data analysis. Design perspectives tie together different phenomena and categories and portray the resulting combination based on the implicitly gained holistic view while roaming through the gathered user research data. Unlike insights or more developed observations noted down as a byproduct during the analysis design perspectives are formed afterwards as a separate task, thus allowing to re-examine the whole of the analyzed data and the analysis process in parallel with the thus far produced deliverables. They are created by sampling individual phenomenon or dominant groups of phenomena within a category or combining items from several categories. The chosen sampling tactics contribute to the nature of the formulated design perspectives as illustrated in figure 1:

- Design perspectives sampled from categories and singular phenomenon tend to outline potential contradictions (design perspective 1).

- Design perspectives local to a category emphasize concurring or supporting tendencies (design perspective 2) or

- When merging two or more categories even potential out-of-scope synergies (design perspective 4). 
- If the sampled phenomena are local to a user or an event (design perspective 3) design perspective can emphasize an observation that would otherwise be lost during the conventional data analysis aiming for generalized consensus opinion.

It is important that the design perspectives are grounded on actual traceable phenomena or categories, thus it is possible to track down the concept candidates' underlying motives and reasoning in later stages of the overall process.

\section{Building the Design Perspectives}

This chapter portrays the process of building the design perspectives. The possible methods and outcomes are illustrated with examples, in italic, from authors' current project in which concepts to support mobile and distributed knowledge work are developed. The analysis framework depicted in figure 1 and definition of entities therein steer the process, but leaves fairly free hands for the implementers regarding the methods and tools used in the analysis. The discussion and suggestions included in the chapter are based on lessons learnt from current and past research projects.

\subsection{Preparation of the Collected Data}

Building the design perspectives begins with preparing the gathered data so that it is 'equally' and easily available for all the members of the analysis team. Strict transcripts are not always necessary, but easily understandable notes and summaries of all the materials are required. Use of highly visual representations can expedite the adoption process, for instance Graphical User Profiles or GUPs [13] have been found useful in cases where user research and concept development has been focused on individual persons. At this point the data should be kept as free as possible of interviewers/observers own interpretations, precognitions and opinions.

In our current project we used interviews and photograph probes (derived from the photography based artefact analysis [14]) as methods in user research. After the field work we produced summaries of interviews and made outlines of photograph probes debriefing interviews. The outlines showed the photographs the users' had taken and the debriefing conversation related to each photograph.

\subsection{Producing Overview of the Collected Data}

Next the information is shared among the analysis team. The thoroughly prepared data is handed out to every member of the analysis team for private reading and familiarizing followed by a group session where the data is walked through and explained. If the documented data is just distributed in a document format absorbing it requires a lot both from the readers and the document, as it is easy to interpret summaries and descriptions differently from its preparer's intentions.

First each researcher read the summaries and outlines by themselves and afterwards we organized a meeting in which the outlines were talked through and found misunderstandings were corrected. Special attention was paid on user's comments and citations so that the context of the citation or comment would not be lost. 


\subsection{Data Analysis}

After developing a shared understanding of the whole gathered data the actual analysis begins. First step is to reorganize and prioritize the observations and other distinct data entities. Associative information management methods such as affinity diagrams [4] are useful and efficient way to reorganize the information. Emerging groups of related observations form phenomena. It is advisable to label phenomena (groups) with mutually understood names and explanations. The phenomena are then further organized or regrouped to categories. It is important to remember that the whole material does not need to be organized under one top category.

We used an applied version of contextual design's affinity diagrams as analysis tool. As a result of organizing and prioritizing the data, categories for enablers and disablers of mobility and distributedness emerged in addition of more traditional categories of user and environment characteristics, different work contexts and work related tasks of mobile workers.

The correctness of categories and phenomena need to be double-checked. At this point it is useful to write down the first full versions of the results (e.g. user profiles, environment descriptions, work flows, etc.). This forces to tie the open ends and check the validity of the results. The final categories and phenomena and relationships between them expose the full complexity and richness of the studied subject in a compact form for the researchers.

\subsection{Creating the Design Perspectives}

At this point it is advisable to take a step backwards and appreciate the effort put into gaining a full exposure to the user research data and compare the results to the objectives of the research and the project. Design perspectives allow incorporating other related information to create more accurate starting points for future product (concept) development.

Some design perspectives may surface directly from the categories as an extension of the analysis. Since the design perspectives are to outline the most interesting events and characteristic of the users and their context of use from the company's and projects' point of view there usually are a couple of candidates already present in the analysts' minds.

More illusive (and more useful) cross-category or single phenomenon design perspectives can be produced by a simple build-and-test method. Examining different categories and phenomena for supporting or contradicting aspects, selecting potentially interesting relationships to form a working hypothesis and than testing what new meanings this sample projects to the earlier gained knowledge. Observing the so far produced results from the users' point of views is another useful way to find interesting and potentially important insight and produce design perspectives.

One design perspective build in our project was a conflict between virtual working environment and real-world work context. Phenomena leading to this design perspective were:

- Computer Supported Collaborative Work (CSCW) tools enabled collaboration between distributed workers via enabling information sharing (e.g. document databases). 
- Team's object of work, renovating an old office building, set numerous requirements and restrictions to team members' work.

- Representing a physical object as complex as a building in a concrete and complete manner was difficult with the available documentation practices.

When creating the design perspectives we realized that the centrality of the object (i.e. the building) in actual work and invisibility of it in virtual working environment produced a conflict and caused numerous problems when using the CSCW tools.

Another design perspective described the different ways the users used the same tools and the different views the users had of the tools. The different point of views and usage policies resulted in misunderstandings of the other parties' intentions.

The last phase is to document all findings, raised questions and blind spots found during the analysis process. These unknown factors need to be reconsidered later during the development and evaluation of the concepts and during the actual product development. In some cases there may even be a need to continue the research phase in order to expose the missing bits and pieces and to understand the findings more deeply.

\section{Conclusions}

Reporting the results of user research in a complete and easily adoptable manner is difficult, as competing viewpoints fragment the information into different documents or different section within a document. This behavior causes problems in a concept development process, as large quantities of analytical conclusions may remain as tacit knowledge inside the analysts' minds. If and when there are changes in the lineup of the development taskforce or the user research is outsourced, these valuable pieces of information are lost from the continuing development phases.

We have observed that even the most scrutinized user and context reports sometimes offer insufficient starting point for successful innovation process since the point of view from which the results are depicted is different from the point of view they are used from.

We suggest design perspectives as a simple and easily adoptable method to alleviate above problems. Design perspectives are an added abstract continuation of conventional user research allowing transition of tacit knowledge from the user research team to the group of people continuing the development process. Our experiences have shown them to better support the special requirements set by usercentered concept development processes, especially the innovation phases.

Design perspectives emphasize the tacit knowledge of the user research team and the point of views of the actual users. They also reflect the results of user research to the overall goals of a project, thus connecting separate sections of information and explicitly stating the otherwise difficult to grasp interconnection within the analyzed data.

In addition design perspectives support tracking decision making during the analysis phase by built-in traceability all the way down to an individual phenomenon. The actual phenomena and even observed events can be traced from the design perspectives. Thus mapping the design perspectives to user profiles and environment descriptions and other presentations useful in concept evaluation phase is effortless. 
Acknowledgments. The authors would like to acknowledge the support from the Intact and Distributed workplace - dWork projects funded by Tekes (National technology agency of Finland) and participating companies for making this study possible. Additionally we would like to express out gratitude to Ms. Laura Turkki for her significant contributions to the initial version of the analysis framework.

\section{References}

1. ISO 13407 International Standard: Human-centred design processes for interactive systems. International Organization for Standardization, Geneve (1999)

2. Millen, D.R.: Rapid Ethnography: Time Deepening Strategies for HCI Field Research. In: Proc. DIS'00, pp. 280-286. ACM Press, New York (2000)

3. Gaver, B., Dunne, T., Pacenti, E.: Design: Cultural Probes. Interactions 6(1), $21-29$ (1999)

4. Beyer, H., Holtzblatt, K.: Contextual Design: Defining Customer-Centered Systems. Academic Press, San Francisco (1998)

5. Hackos, J.T., Redish, J.C.: User and Task Analysis for Interface Design. John Wiley \& Sons, New York (1998)

6. Kuniavsky, M.: Observing The User Experience: A Practitioner's Guide to User Research. Morgan Kaufmann Publishers, San Francisco (2003)

7. Creswell, J.W.: Qualitative Inquiry and Research design: Choosing Among Five Traditions. SAGE Publications, London (1998)

8. Collier, J., Collier, M.: Visual Anthropology: Photography as Research Method. University of New Mexico Press, Albuquerque (1986)

9. Fettermann, D.M.: Ethnography Step by step. SAGE Publications, London (1989)

10. Salovaara, A., Mannonen, P.: Use of Future-Oriented Information in User-centered Product Concept Ideation. In: Costabile, M.F., Paternó, F. (eds.) INTERACT 2005. LNCS, vol. 3585, pp. 727-740. Springer, Heidelberg (2005)

11. Nieminen, M.P., Mannonen, P.: User-centered Product Concept Development. In: International Encyclopedia of Ergonomics and Human Factors, 2nd edn. pp. 1728-1732. Taylor \& Franciss, New York (2005)

12. Schön, D.A., Rein, M.: Frame Reflection: Toward the Resolution of Intractable Policy Controversies. Basic Books, New York (1994)

13. Kankainen, T., Parkkinen, J.: GUP: graphical presentation of user profile. In: CHI '01 extended abstracts on Human factors in computing systems, pp. 105-106. ACM Press, New York (2001)

14. Mannonen, P., Kuoppala, H., Nieminen, M.P.: Photography Based Artefact Analysis. In: Proc. INTERACT'03, pp. 833-836. IOS Press, Amsterdam (2003) 